\title{
Aplicando Flipped Teaching en Física del Grado en Ingenieria de Sistemas de Telecomunicación, Sonido e Imagen
}

\author{
Jesús Alba Fernández ${ }^{\mathrm{a}}$, Constantino Torregrosa Cabanilles ${ }^{\mathrm{b}}$, Anna Vidal Meló ${ }^{\mathrm{c}}$ y \\ Romina del Rey Tormos ${ }^{\mathrm{d}}$ \\ ajesalba@fis.upv.es, ${ }^{\text {b }}$ ctorregr@fis.upv.es, ${ }^{\text {cavidal@mat.upv.es, }, \text { roderey@upvnet.upv.es }}$
}

Grup d'Innovació Educativa i recerca en Matèries Científiques (GIERMAC)

EPSG. Escola Politècnica Superior de Gandía. Departament de Física Aplicada i Departement de Matemàtica Aplicada. EPSA. Escola Politècnica Superior d'Alcoi. Departament de Física Aplicada.

\begin{abstract}
In November 2016, the GIERMAC team (Grup d'Innovación Educativa $i$ Recerca en Matèries Cientifiques) received a PIME-UPV project. This project was entitled "Startup of several experiences with the Flip Teaching approach in Physics and Mathematics subjects" and it is a continuation of a previous project entitled "Study on the application of Flipped Teaching in Mathematics and Physics subjects". This paper presents the application of Flip Teaching during two academic years in the subject of Physics of the Bachelor's Degree in Engineering of Telecommunications Systems, Sound and Image (GISTSI). Experience focuses on thermodynamics items. Students present in volunteer way and are compensated with additional points for strive.
\end{abstract}

Keywords: Flippled Teaching, class reverse, physics, thermodynamics, project-based learning.

\footnotetext{
Resumen

Al "Grup d'Innovación Educativa i Recerca en Matèries Cientifiques (GIERMAC)", se le concedió en noviembre de 2016 el proyecto PIME-UPV, "Puesta en marcha de diversas experiencias con el enfoche Flip Teaching en asignaturas de Física y matemáticas", continuación de otro proyecto anterior titulado "Estudio sobre la aplicación del Flip Teaching en asignaturas de Matemáticas y Física". En el trabajo se presenta la aplicación del Flip Teaching en dos cursos académicos en la asignatura de Fisica del Grado en Ingeniería de Sistemas de Telecomunicación, Sonido e Imagen (GISTSI). La experiencia se centra en temas de termodinámica a la que los alumnos se presentan de forma voluntaria y son compensados con puntos adicionales por el esfuerzo.
} 
Palabras clave: Flippled Teaching, clase inversa, Física, Termodinamica, Aprendizaje basado en proyectos

\section{Introducción}

En noviembre de 2016 se concede al "Grup d'Innovación Educativa i Recerca en Matèries Científiques (GIERMAC)" el proyecto PIME-UPV "Puesta en marcha de diversas experiencias con el enfoche Flip Teaching en asignaturas de Física y matemáticas", continuación de otro proyecto anterior titulado"Estudio sobre la aplicación del Flip Teaching en asignaturas de Matemáticas y Física”. En el primer proyecto se presentó ya una primera experiencia completa realizada con los alumnos de Física del Grado en Ingeniería de Sistemas de Telecomunicación, Sonido e Imagen (GISTSI) durante los meses de noviembre y diciembre de 2015 y en esta continuación se analiza la evolución en los dos cursos (2015-2016 y 2016-2017).

El modelo pedagógico Flip Teaching o Clase Inversa envía el trabajo de determinados procesos de aprendizaje fuera del aula, y usa el tiempo de clase para potenciar procesos más prácticos. Suele ser común que el alumno no reciba la tradicional clase de pizarra y/o transparencias de materias concretas, puesto que en esta metodología se supone que lo hará el alumno fuera de clase con materiales, documentación e información preparados con antelación por el profesor (Bergamm, 2005; Lage, 2000; Talbert, 2012a; Talbert, 2012b; Moravec, 2010; Gannod, 2008; Talbert 2014). La Universitat Politécnica de Valencia tiene un programa específico que apoya los proyectos piloto basados en Flip Teaching, lo que da idea de la importancia que se le está dando al modelo.

Para facilitar el proceso de aprendizaje fuera del aula, dentro del proyecto PIME-UPV citado se están preparando una serie de fichas de recursos con la siguiente información:

1. Datos Básicos de la Asignatura a la que se aplicará: Asignatura o asignaturas a las que afecta y ámbito

2. Tema-Sección: Qué temas/materiales son los que se trabajan con esta metodología

3. Objetivo de Aprendizaje: al finalizar la actividad FLIP, qué será capaz el alumnado.

4. Actividad Flip: qué tipo de metodologías se deciden, programación, etc.

5. Recursos y Descripción: de qué materiales van a disponer los alumnos

6. Evaluación: que áctos evaluativos se consideran

En el caso concreto de Física, se aplica en el primer semestre, durante los meses de noviembre y diciembre, esta metodología a temas de Termodinámica (Serway y Jewett, 2005 ; Serway y Jewett, 2008 ; Tipler, 1992 ; Tipler, 1994). Para ello se prepara la ficha correspondiente, y se desarrolla una metodología basada en el aprendizaje basado en proyectos (PBLs) de carácter voluntario como en (Alba et al, 2015a; Alba et al, 2015b) (Alba et al, 2016) y cuya evaluación supone un premio adicional al alumno, de viene a ser equivalente a subir medio punto la nota final de la asignatura.

(cc) EY-NC-ND 2017, Universitat Politècnica de València 


\section{Objetivos}

El objetivo principal es asentar la metodología de clase inversa en la asignatura FISICA del Grado en Ingeniería de Sistemas de Telecomunicaciones, Sonido e Imagen (GISTSI), para contrarestar la desmotivación en los alumnos que la enseñanza más clásica en esta asignatura puede producir. Para ello se prepara la ficha de actuación y recursos que forma parte del proyecto PIME-UPV citado.

La asignatura FISICA es una asignatura anual fundamental de primer curso. En el primer semestre se tratan las siguientes unidades didácticas:

1. Leyes del movimiento

2. Energía

3. Momento

4. Oscilaciones

5. Ondas

6. Temperatura y calor

7. La segunda ley

Estos siete bloques temáticos deben impartirse en 15 semanas de clase en el primer semestre. Además realizan 3 prácticas de 2 horas durante el dicho semestre. Por tanto, el alumno tiene 45 horas presenciales en el citado semestre y 67,5 no presenciales realizando el cómputo de horas según créditos.

En el caso que nos ocupa se decide trabajar con las unidades temáticas "6. Temperatura y Calor" y "7. La Segunda Ley". Para ponerla en práctica se propone una metodología basada en PBLs de termodinámica, ya que parece que el PBL es recomendable en grados de ingeniería (Aparicio et al, 2005 ; Case y Light 2011 ; Calvo et al, 2010 ; De Miguel, 2006).

Se plantean los siguientes objetivos de aprendizaje. El alumno al finalizar la actividad FLIP debe ser capaz de:

- Explicar las diferencias entre calor y temperatura

- Explicar las diferentes escalas termométricas y tipos de termómetros

- Formular con expresiones matemáticas con los efectos del calor

- Identificar la primera Ley de la Termodinámica

- Formular la transmisión de energía a través de la conducción, convección y radiación.

- Explicar el concepto de máquina térmica

- Identificar los ciclos conocidos (Carnot y Otto)

- Identificar y formular la segunda ley de la termodinámica

\section{Desarrollo de la Innovación}

Como se ha comentado, se plantea la actividad flip en base a PBLs de los dos temas de termodinámica de la asignatura Física. Se ha creado una ficha-recurso en base a los puntos 
citados en la parte de introducción. La primera versión de la ficha se usó en el curso 20152016 y los resultados se mostraron en el In-red 2016 (Alba et al, 2016). La segunda versión de la ficha, aplicada en el curso 2016-2017 es la que se presenta a continuación. 


\begin{tabular}{|c|c|}
\hline $\begin{array}{c}\text { PROYECTO DE INNOVACIÓN Y MEJORA EDUCATIVA } \\
\text { CURSO 2016-2017 } \\
\text { UPV }\end{array}$ & \begin{tabular}{c} 
Estudio sobre la aplicación del Flip Teaching en asignaturas de Matemáticas y Fisica \\
\hline
\end{tabular} \\
\hline
\end{tabular}

\section{DATOS BASICOS DE LA ASIGNATURA}

Nombre: FISICA. Código: 11264

Grado en Ingenieria de Sistemas de Telecomunicación, Sonido e Imagen. Carácter: Formación básica Créditos: 9,00 --Teoría: 4,5 --Prácticas: 4,5

\section{TEMA-SECCIÓN \\ Temas 6 y 7. Termodinámica: Temperatura y Calor. La segunda Ley. \\ Proyecto sobre termodinámica}

3. OBJETIVOS DE APRENDIZAJE
Al finalizar la actividad FLIP propuesta, el alumnado será capaz de:
1. Explicar las diferencias entre calor y temperatura
2. Explicar las diferentes escalas termométricas y tipos de termómetros
3. Formular con expresiones matemáticas con los efectos del calor
4. Identificar la primera Ley de la Termodinámica
5. Formular la transmisión de energía a través de la conducción, convección y radiación.
6. Explicar el concepto de máquina térmica
7. Identificar los ciclos conocidos (Carnot y Otto)
8. Identificar y formular la segunda ley de la termodinámica

\section{ACTIVIDAD FLIP}

Realización de proyectos de los dos temas. Se asigna un proyecto por defecto pero los grupos pueden elegir temas alternativos. La descripción del proyecto básico es la siguiente:

TITULO DEL PROYECTO: Fabricación y calibración de un termómetro casero.

ENUNCIADO: Diseñar y fabricar un termómetro casero con cierta calibración. Debe permitir ciertos rangos de temperatura.

PREGUNTA MOTRIZ ¿Todos los termómetros son de mercurio?

PLANIFICACIÓN: 4 semanas

Semana 1: Búsqueda de información y lectura de materiales.

Semana 2: Trabajo en grupo. Entregable 1: Descripción del posible prototipo y materiales a utilizar. Respuesta a la pregunta motriz. Previsión de cálculos teóricos del prototipo.

Semana 3: Trabajo en grupo. Avance de borrador de poster, presentación o audiovisual. Semana 4: Presentación del poster o audiovisual y de los prototipos en clase.

5. RECURSOS Y DESCRIPCIÓN

En la descripción del PBL básico se recomiendan los siguientes videos de construcción de termómetros: 


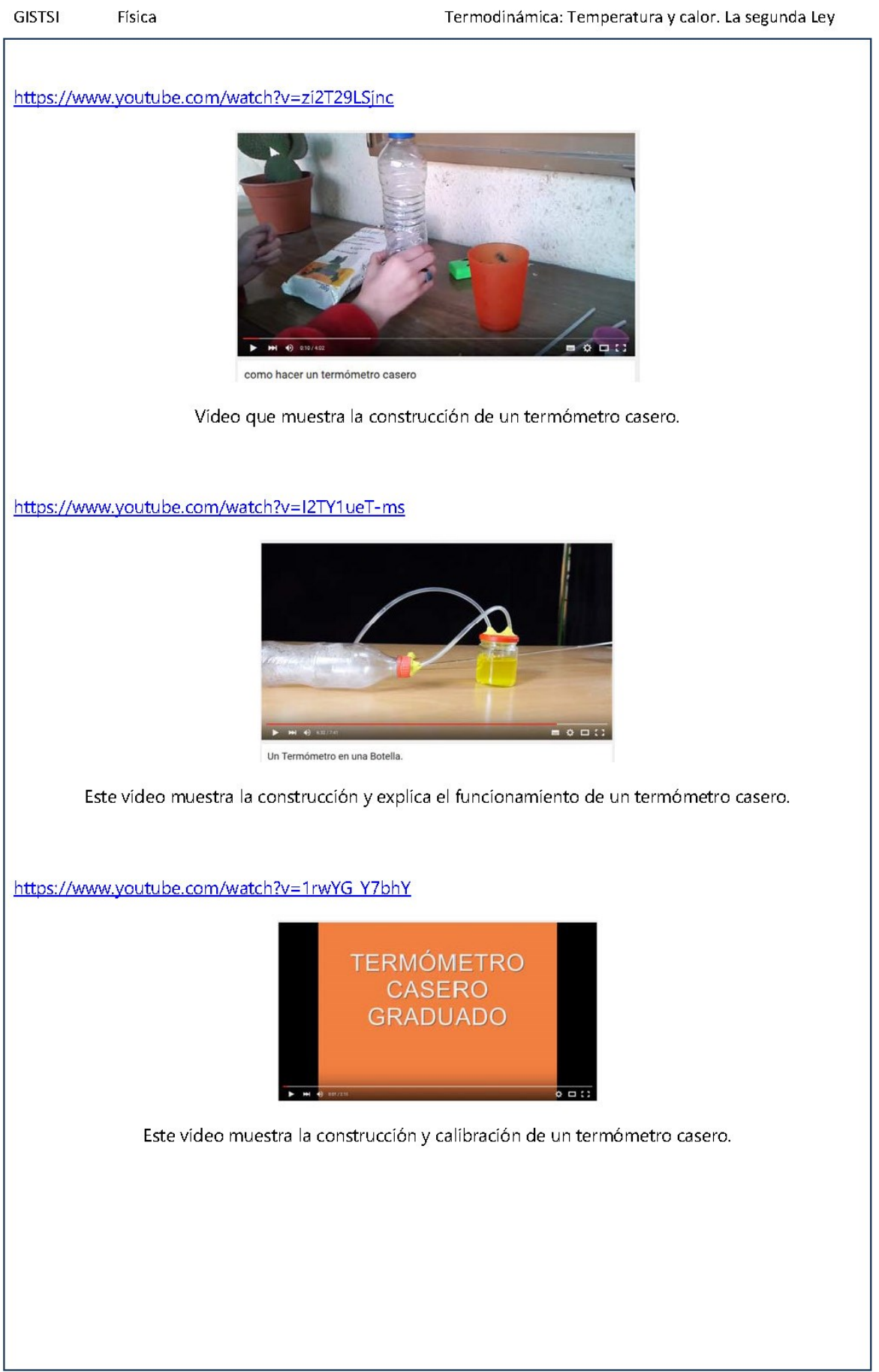

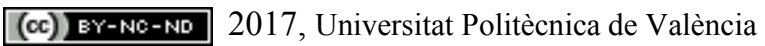

Congreso IN-RED (2017) 


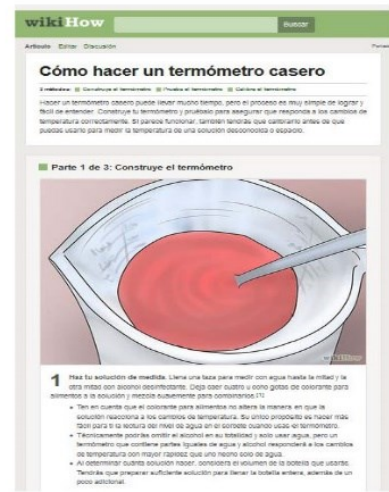

6. OTROS RECURSOS Y DESCRIPCIÓN

Además de la documentación del PBL básico sobre el termómetro, se tienen los siguientes recursos:

* Transparencias resumen de los temas 6 "Calor y temperatura" y 7 "La segunda Ley".

* Boletín de problemas de los dos temas

* Memoria de práctica sobre los dos temas "interpretación estadística de la entropía"

* Videos de trabajos realizados

* Páginas web con trabajos y experimentos resueltos

\section{EVALUACIÓN}

Se valoran 20 puntos (sobre 400 puntos) en tres partes: el entregable 1, el poster o similar y la presentación pública. Para la presentación se prepara rúbrica de profesores y alumnos.

Para ponerla en práctica se realiza una planificación de cinco semanas, que para el curso 2016-2017 puede verse en la tabla 1.

Tabla 1. PLANIFICACION del PBL

\begin{tabular}{|l|l|l|}
\cline { 2 - 3 } \multicolumn{1}{l|}{} & SEMANA & TAREAS \\
\hline Semana 1 & $14 / 11 / 16-18 / 11 / 16$ & $\begin{array}{l}\text { Búsqueda de información. Lectura de materiales. } \\
\text { Entrega de hoja firmada }\end{array}$ \\
\hline Semana 2 & $21 / 11 / 16-25 / 11 / 16$ & Trabajo en grupo \\
\hline Semana 3 & $28 / 11 / 16-02 / 12 / 16$ & Trabajo en grupo. Entregable 1 \\
\hline Semana 4 & $05 / 12 / 16-10 / 12 / 16$ & $\begin{array}{l}\text { Trabajo en grupo. Avance de borrador de poster o } \\
\text { similar. }\end{array}$ \\
\hline Semana 5 & $12 / 12 / 16-16 / 12 / 16$ & Problema de clase 3 y Test 3.Presentación de trabajos. \\
\hline
\end{tabular}

Entregable 1. 02/12/16. Entrega de la descripción del posible prototipo y materiales a utilizar. Previsión de cálculos teóricos del prototipo.

POSTER. 10/12/16. Avance del póster o similar 
Problema 3 y Test 3. 12/12/16. Problema de clase 3 y test 3(MAS y ondas). Avance del póster o similar

Presentación. 16/12/16. Todos los miembros del grupo presentan su prototipo en clase. Todos los entregables y la presentación son de grupo. Como en el curso 2015-2016 (Alba et al, 2016) varios grupos decidieron trabajar otros proyectos distintos (hornos solares, coche a vapor, barco pop-pop, generador eléctrico, Stirling, cohete, etc.).

La asignatura Fisica se evalúa sobre 400 puntos (400 puntos se corresponde con el 10) con diferentes actos evaluativos que van acumulando puntos. Esta actividad se propone como premio adicional con 20 puntos (sobre 400 puntos) en tres partes: el entregable 1, el poster o similar y la presentación. Para la presentación se prepara rúbrica de profesores y alumnos. En otros actos evaluativos (parcial de enero) los temas de termodinámica también se evalúan en preguntas tipo test.

Se prepara una rúbrica única para profesores y alumnos en el curso 2016-2017. Esto se ha modificado y simplificado respecto al curso anterior, donde se usan dos rúbricas diferentes donde el lenguaje no facilitaba cuplimentarlas. Por ello se ha optado en una simplificación que facilite y agilice la coevaluación.

Tabla 2. Rúbrica de coevaluación

\begin{tabular}{|l|l|l|l|l|}
\hline Competencias & GR-1 & GR-2 & GR-3 & GR-4 \\
\hline 1) Es capaz de diseñar el equipo/objeto & & & & \\
\hline 2) Define los objetivos y las variables del trabajo & & & & \\
\hline 3) Resolución del problema & & & & \\
\hline 4) Mecánica de la presentación & & & & \\
\hline 5) Póster/material de presentación & & & & \\
\hline NOTA GLOBAL PROPUESTA (0 A 10) & & & & \\
\hline
\end{tabular}

Como novedad, en el curso 2016-2017 se incorpora la competencia transversal de "comunicación oral efectiva", como se había señalado en un trabajo anterior (Alba et al, 2016). Las presentaciones de los proyectos se presentaron tras la asistencia de los alumnos a un curso del ICE sobre Comunicación Efectiva, con el objetivo de que los alumnos aprendan a expresar ideas de forma estructurada e inteligible en presentaciones orales de duración breve o en intervenciones puntuales.

Se solicita a los alumnos que en el proyecto apliquen lo aprendido en el curso para la presentación de los proyectos. Además se utilizó para evaluar las presentaciones la rúbrica UPV CT-08 sobre comunicación efectiva, en la dimensión de comunicación oral y el nivel de dominio I.

(cc) EY-NC-ND 2017, Universitat Politècnica de València 


\section{Resultados}

En el curso 2015-2016 se acogieron a la primera experiencia 33 alumnos de 53 en equipos de 3 o 4 alumnos (un 62\%). Como curiosidad, la mayoría de repetidores de la asignatura no realizaron la actividad (Alba et al, 2016). Respecto a las notas, todas estaban entre 8 y 10 puntos obtenidas combinando las rúbricas de alumnos y profesores.

En el curso 2016 - 2017 se presentaron inicialmente 10 proyectos en grupos de 2 a 4 estudiantes, de los cuales 8 llegaron a término: un termómetro casero, un horno solar, un coche a vapor, dos barcos a vapor, un motor stirling, un cohete y un generador eléctrico a vapor. Participaron 28 de los 48 alumnos de la assignatura (58\%). Los porcentajes de alumnos que deciden de forma voluntaria realizar la actividad. Las notas, como resultado del promedio de notas asignadas por los propios alumnos a cada proyecto, en base a una sencilla rúbrica de evaluación, oscilaron entre 7.5 y 8.7 puntos sobre 10 (media 8.1 y desv 0.5 ), con desviaciones estándar de cada nota entre 0.8 y 1.3 puntos. A continuación se muestran algunas fotografías de la edición 2016-2017.
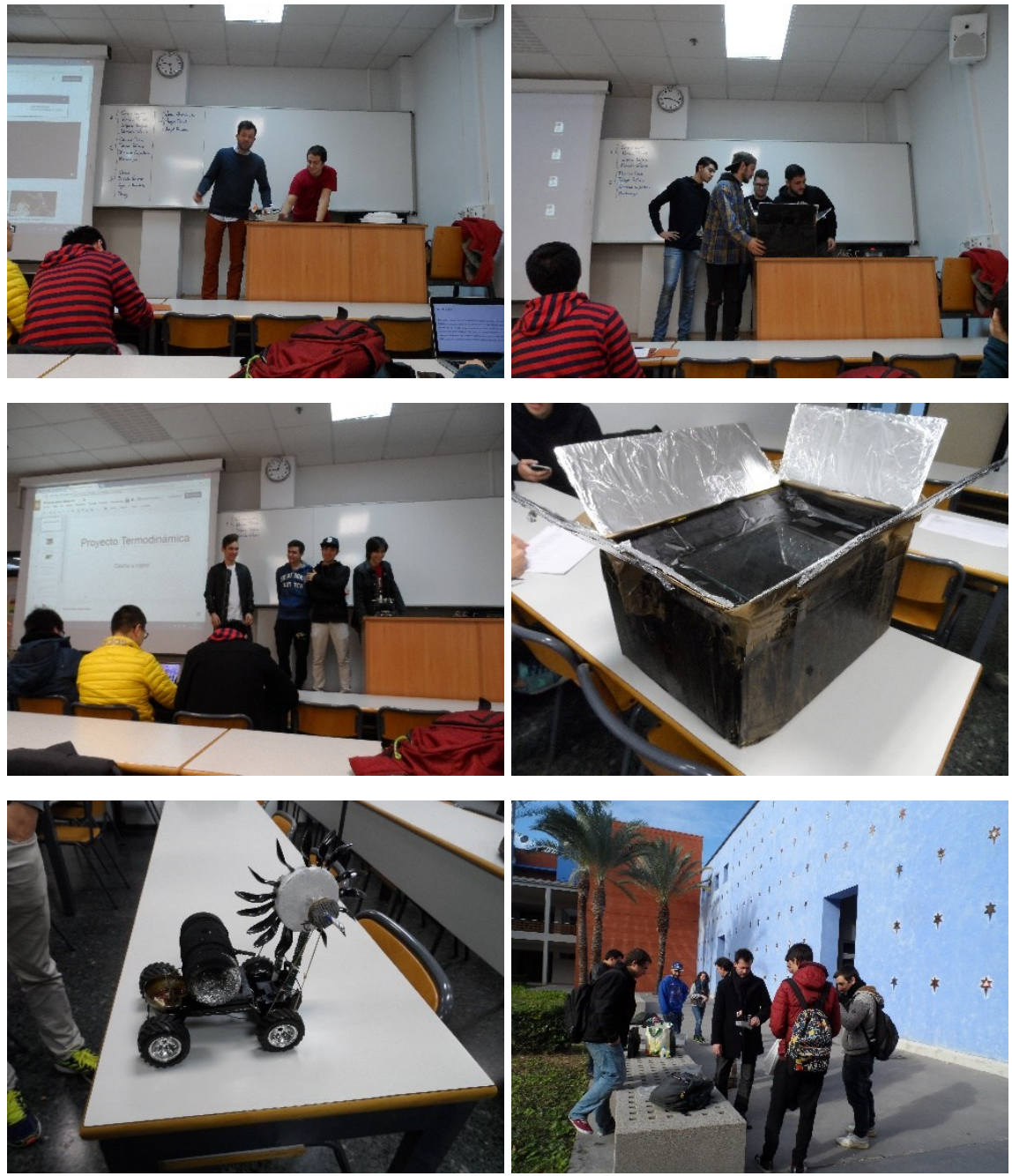

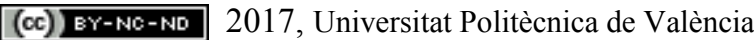

Congreso In-Red (2017) 

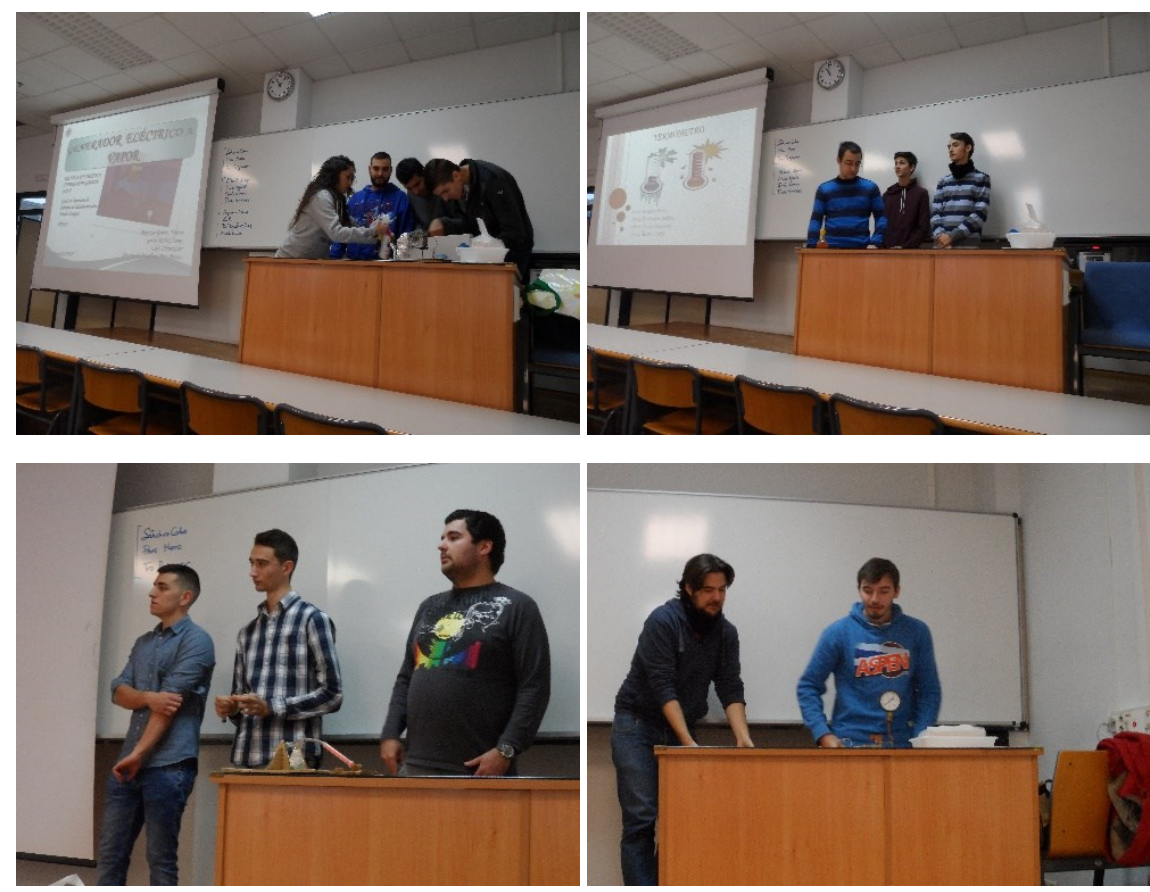

\section{Conclusiones}

En este trabajo se ha mostrado un resumen de la experiencia de poner en marcha y asentar la metodología Flip Teaching en la asignatura Física del Grado en Ingenieria de Sistemas de Telecomunicación, Sonido e Imagen. La previsión para el próximo curso es seguir manteniendo esta actividad.

Se realiza una revisión sobre fortalezas, debilidades, amenazas y oportunidades respecto a a la aplicación de esta metodología.

Las fortalezas observadas en los dos cursos son las siguientes:

- Se reactiva la asistencia a clase. La experiencia muestra un aumento claro de la asistencia.

- La elección de múltiples opciones de proyectos refuerza la confianza del alumno.

- Se potencia el trabajo en grupo y la comunicación oral efectiva, que se trabaja como competencia transversal.

- Los mismos alumnos pueden crear materiales para usar en cursos siguientes. Ya se dispone material de dos cursos.

- Se aumenta la motivación del alumnado

Las debilidades actuales: 
- La carga de trabajo de alumnos y profesores es mucho mayor. Se calcula que se ha triplicado el número de horas de faena en el primer año de implantación, aunque se ha rebajado algo el segundo año.

- El ritmo de los trabajos no es fluido. Viene afectado por festivos y sobre todo por interferencias de otras asignaturas con evaluaciones clásicas o tradicionales.

- Hay que elegir bien los contenidos. Hay material erróneos o con inexactitudes en las redes.

Las amenazas detectadas:

- No existe una apuesta real por este tipo de metodologías por parte de la universidad, aunque el mensaje sea el contrario. Un ejemplo claro: para el curso 2016-2017 se redujeron los grupos de prácticas de la asignatura. Al mismo tiempo que se nos anima a realizar este tipo de iniciativas, nos recortan los recursos para realizarlas.

- Hay que limitar los proyectos puesto que algunos pueden tener riesgos, creando un protocolo acorde.

- Los alumnos pueden llegar a pensar que no se ha impartido el tema correspondiente.

Existe una oportunidad clara de centrar el tiempo de aula en cuestiones más prácticas, pero la actual manera de asignar la carga docente no contempla este tipo de iniciativas.

\section{Agradecimientos}

Este trabajo se ha realizado gracias al apoyo del Vicerrectorado de Estudios, Calidad y Acreditación de la UPV al Proyecto PIME 2016-2017 "Puesta en marcha de diversas experiencias con el enfoque Flipped Teaching en asignaturas de Matemáticas y Física".

\section{Referencias}

ALBA J., DEL REY, R., VIDAL A., ROIG B. (2015a) Aprendizaje Basado en Proyectos en el Grado en Ingeniería de Sistemas de Telecomunicaciones, Sonido e Imagen. Caso práctico del electroscópio como experiencia interdisciplinar entre Física y Matemáticas. 23 Congreso Universitario de Innovación Educativa de las Enseñanzas Técnicas (XXIII CUIEET). Valencia 15-17 de julio de 2015.

ALBA J., TORREGROSA C., DEL REY R. (2015b) Aprendizaje basado en proyectos: Primera experiencia en la asignatura de Física del Grado en Ingeniería de Telecomunicación, Sonido e Imagen. Universitat Politècnica de València Congreso IN-RED (2015)

ALBA J., TORREGROSA C., VIDAL A., DEL REY R. (2016) Flipped Teaching en Física del Grado en Ingenieria de Sistemas de Telecomunicación, Sonido e Imagen: primeros resultados. Universitat Politècnica de València Congreso IN-RED (2016)

(c)) EY-NC-ND 2017, Universitat Politècnica de València

Congreso In-Red (2017) 
PARICIO, F., GONZALEZ, R. M. Y SOBREVILA, M. A. (2005). Formación de Ingenieros. Objetivos, métodos y estrategias. Instituto de Ciencias de la Educación, UPM.

BERGMANN, J., SAMS, A. (2015). Dale la vuelta a tu clase: Lleva tu clase a cada estudiante, en cualquier momento y cualquier lugar. SM.

CASE, J. M. Y LIGHT, G. (2011). Emerging Methodologies in Engineering Education Research. Journal of Engineering Education, 100 (1), 186-210.

CALVO, I., LOPEZ-GUEDE, J.M. Y ZULUETA, E. (2010). Aplicando la metodología Project Based Learning en la docencia de Ingeniería Técnica en Informática de Gestión, Revista de Formación e Innovación Educativa Universitaria. Vol. 3, No 4, 166-181

DE MIGUEL, M. (2006). Modalidades de Enseñanza centradas en el desarrollo de competencias. Universidad de Oviedo.

GANNOD, G., BURGE, J., HELMICK, M. (2008). "Using the inverted classroom to teachsoftware engineering" en Proceedings of the International Conference on Software Engineering (ICSE). Leipzig, Germany. p. 10-18

LAGE, M.J., PLATT, G.J., TREGLIA, M. (2000). "Inverting the classroom: A gateway to creating an inclusive learning environment" en The Journal of Economic Education, vol. 31 , issue 1 , p. $30-43$

MORAVECD, M., WILliAMS, A., AGUILAR-ROCA, N., O’DOWD, D.K. (2010). "Learn before lecture: a strategy that improves learning outcomes in a large introductory biology clas" en CBE Life Sci Educ, vol. 9, p. 473-481

SERWAY, R. A. Y JEWETT, J. W. (2005). Física para ciencias e ingeniería. Volumen 1. México D.F. International Thomson, $6^{\mathrm{a}}$ ed.

SERWAY, R. A. Y JEWETT, J. W. (2008). Física para ciencias e ingenierías. Volumen II. México D.F. : Cengage Learning.

TALBERT, R. (2012a). "Learning MATLAB in the Inverted classroom" en Proceedings of the ASEE Annual Conference. San Antonio, Texas. 25.883.1-25.883

TALBERT, R. (2012b). “Inverted Classroom” en Colleagues, vol. 9, issue 1, article 7

TALBERT, R. (2014). "Inverting the Linear Algebra Classroom" en PRIMUS (Problems, Resources, and Issues in Mathematics Undergraduate Studies), vol. 24, issue 5, p. 361-374

TIPLER, P. A. (1992) Física Tomo 1. Barcelona etc. : Reverté

TIPLER, P. A. (1994) Física Tomo 2. Barcelona etc. : Reverté

UPV, UNIVERSITAT POLITECNICA DE VALENCIA. Competencias transversales UPV $<$ http://competenciast.webs.upv.es/> [Consulta: 2 de abril de 2016]

VILLA, A. Y POBLETE, M. (2007): Aprendizaje basado en competencias. Una propuesta para la evaluación de las competencias genéricas. Ediciones Mensajero. Bilbao. 